\title{
Radial Basis Function and K-Nearest Neighbor Classifiers for Studying Heart Rate Signals during Meditation
}

\author{
Ateke Goshvarpour \\ Department of Biomedical Engineering, Mashhad Branch, Islamic Azad University, Mashhad, Iran. \\ E-mail: ateke.goshvarpour@gmail.com \\ Atefeh Goshvarpour* \\ Department of Biomedical Engineering, Mashhad Branch, Islamic Azad University, Mashhad, Iran. \\ E-mail: atefeh.goshvarpour@gmail.com
}

\begin{abstract}
Meditation refers to a family of selfregulation practices that focus on training attention and awareness in order to bring mental processes under greater voluntary control. The aim of this study is to evaluate the efficiency of two different classifiers, kNearest Neighbor (k-NN) and Radial Basis Function (RBF), on the heart rate signals in a specific psychological state. For this purpose, two types of heart rate time series (before, and during meditation) of 25 healthy women are collected in the meditation clinic in Mashhad. The spectral parameters are used to gain insight into the autonomic nervous system (ANS) response induced by meditation. Therefore, very low frequency, low frequency, high frequency, the LF/HF ratio and frequency of the highest spectral peak of heart rate signals are extracted and used as inputs of the classifiers. To evaluate performance of the classifiers, the classification accuracies and mean square error (MSE) of the classifiers were examined. The classification results of this study denote that the RBF classifier trained on spectral features obtains higher accuracy than that of k-NN classifier. The total classification accuracy of the RBF classifier is $92.3 \%$ with 0.026 classification error. However, k-Nearest Neighbor classifier gives encouraging results $(86.5 \%)$. Experimental results verify that radial basis function is an efficient classifier for classifying heart rate signals in a specific psychological state.
\end{abstract}

Index Terms-Frequency Indices, Heart Rate Signals, K-Nearest Neighbor, Meditation, Radial Basis Functions

\section{INTRODUCTION}

Meditation refers to a family of self-regulation practices that focus on training attention and awareness in order to bring mental processes under greater voluntary control and thereby foster general mental well being and development and/or specific capacities such as calm, clarity, and concentration [1]. Meditation is practiced by many to facilitate their health and adaptation to medical illness, as a form of systematic training in a self-regulatory approach to stress reduction and emotion management [2-5].

Heart rate variability analysis has become an important tool in cardiology, because its measurements are noninvasive and easy to perform, have relatively good reproducibility and provide the great information; information was found directly linked to health [6] and prognostic information on patients with heart disease [7].

Different measures of heart rate signals are designed to reflect different aspects of these signals and thus do not always provide the same information about the cardiovascular system [6-10]. Thus, some measures of heart rate signals may be more attuned to the effects of meditation on the cardiovascular system than others.

Literature on cardiac variability conventionally differentiates three bands of cardiac variability: high frequency waves $(0.15-0.4 \mathrm{~Hz})$, low frequency waves $(0.05-0.15 \mathrm{~Hz})$, and very low frequency waves $(0.005-$ $0.05 \mathrm{~Hz})$ [11-13].

The high frequency (HF) waves usually coincide with respiratory sinus arrhythmia (RSA), the increase and decrease in heart rate (HR) produced by the respiratory cycle [14]. RSA is produced by a combination of respiration-induced biochemical changes, changes in intrathoracic pressure, and central vagal stimulation [15-16]. In other words, the high frequency power is widely accepted as a marker of cardiac parasympathetic control. Thus it is often used as a noninvasive means of studying the autonomic balance or, more specifically, the vagal tone of individuals.

Some studies indicate that the low frequency (LF) peak or the ratio of low to high frequency may be an adequate reflection of sympathetic activity [17]; while others showed that low frequency waves are affected by both the sympathetic and parasympathetic systems. They are correlated with baroreflex gain [18], although other rhythms also are closely related to baroreflex activity [19].

Very low frequency (VLF) wave activity is less 
well understood. There is some evidence that it is related to regulation of vascular tone and body temperature [20]. Thus, cardiac variability reflects a variety of homeostatic functions that are specific for particular frequency ranges. Greater amplitude and complexity suggest a greater variety of more active homeostatic reflexes and thus may be indexes of adaptive capacity.

The LF/HF ratio, an estimate of sympathovagal balance, was also calculated from the absolute power of both frequency components [13].

A large number of studies aimed at studying observed effects of meditation and were only able to assess certain general changes of the heart rate signals $[6,11,19]$. However, there is no significant effort on classifying biological signals during rest and meditation.

In this study, heart rate classification algorithm can be divided into three steps: data collection, feature extraction, and classification. Fig. 1 demonstrates the framework of the proposed method. It involves the stages of data collection, feature extraction and classification. Five spectral indices (very low frequency, low frequency, high frequency, the LF/HF ratio and frequency of the highest spectral peak) of heart rate signals of twenty five women referred to meditation clinic are extracted before and during meditation. For classification of these two states (before and during meditation), two different methods are proposed: kNearest Neighbor (k-NN) and Radial Basis Function (RBF). These steps are discussed in more detail in the following section.

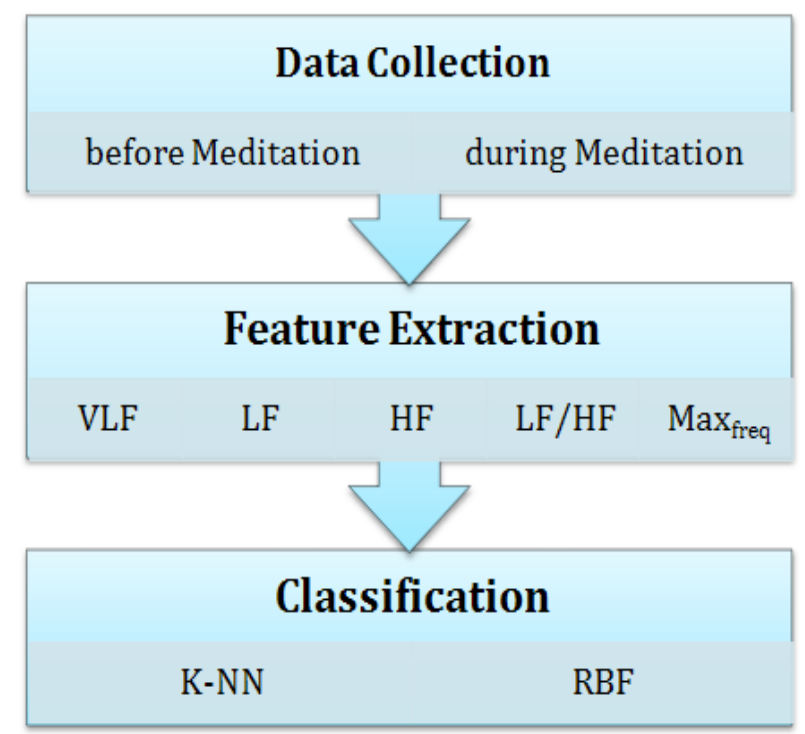

Figure 1. A flow chart of our integrated method. Note:

VLF: very low frequency; LF: low frequency; HF: high frequency; Max ${ }_{\text {freq: }}$ frequency of the highest spectral peak; K-NN: k-nearest neighbor; RBF: Radial basis functions

The outline of this study is as follows. In the next section, we briefly describe the set of heart rate signals (HR) used in our study. Then, the computation of the features (very low frequency; low frequency; high frequency, the LF/HF ratio and frequency of the highest spectral peak of heart rate signals) of HR signals is explained in order to define the behavior of the signal under study. Next, the description of different classifier models that are considered in this study is presented. Finally, the results of present study are shown and the study is concluded.

\section{METHODS}

\section{A. Data collection}

Twenty five subjects took part in the study. Fifteen subjects: eleven meditators (mean age $40.18 \pm 7.19$, mean meditation experience 5 to 7 years) and four nonmeditators (mean age $25.5 \pm 1.91$ ) were asked to do meditation by listening to the guidance of the master. The other ten subjects were asked to do meditation by themselves. They were considered to be at an advanced level of meditation training (mean meditation experience 7 years, mean age $37.8 \pm 6.39$ ).

The subjects were in good general health and did not follow any specific heart diseases. The subjects were asked not to eat salty or fat foods before meditation practices or data recording. Informed written consent was obtained from each subject after the experimental procedures had been explained [21].

The experimental procedure was divided into two different stages: Subjects were first instructed to sit quietly for 5 minutes and kept their eyes closed. After that, they performed meditation. Meditation prescribes a certain bodily posture. They sit on a cushion 5 to 10 centimeters thick that is placed on blanket. They cross their legs so that one foot rests on the opposite thigh with the sole of their foot turned up and with their knees touching the blanket (lotus or half-lotus position). The torso should be kept straight, but it should not be strained. The head should be kept high with eyes closed. During this session, the meditators sat quietly, listening to the guidance of the physician and focusing on the breath [21-22].

The electrocardiogram signals (ECG) - lead I- of all subjects were recorded in meditation clinic using 16channel PowerLab (manufactured by ADInstruments) (Fig. 2).

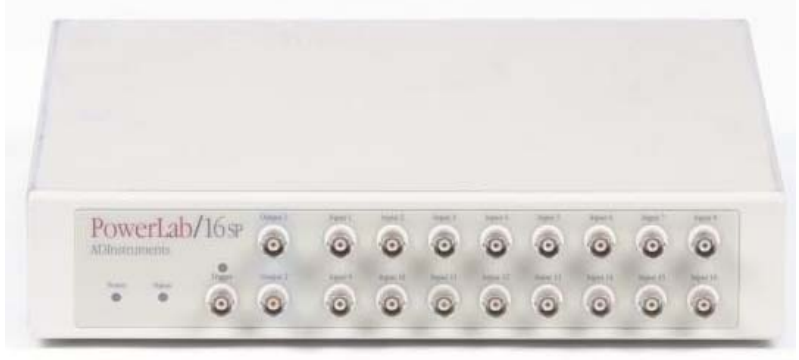

Figure 2. 16-channel PowerLab (manufactured by ADInstruments). 
Heart rate signals were extracted online using Chart5 for Windows software (based on heart rate $=$ $60 /$ RR interval in seconds). The monitoring system hardware filters band passed data in range $0.1-200 \mathrm{~Hz}$ for ECG time series. A digital notch filter was applied to the data at $50 \mathrm{~Hz}$ to remove any artifacts caused by alternating current line noise. The sampling rate was 400 $\mathrm{Hz}$ [21-22].

\section{B. Feature extraction}

The RR interval data were analyzed in terms of HRV parameters in frequency domain. Then, the HRV measures in the meditation and in the rest were compared. The spectral parameters are used to gain insight into the autonomic nervous system (ANS) response induced by meditation.

The spectral HRV measures for each segment were calculated by using Fast Fourier transform [23]. According to the Task Force [13], the power spectrum for short time series can be classified into 3 ranges as follows:

(i) Power in the very low frequency range (VLF), $0.005-0.05 \mathrm{~Hz}$,

(ii) Power in the low frequency range (LF), 0.05-0.15 $\mathrm{Hz}$,

(iii) Power in the high frequency range (HF), 0.15 $0.4 \mathrm{~Hz}$.

\section{Classification}

In this paper, very low frequency; low frequency; high frequency, the LF/HF ratio and frequency of the highest spectral peak of heart rate signals are used as inputs of two different classifiers. The task at hand is to classify the HR epochs of twenty five healthy women as either related to before or to during meditation.

The correct classification rate not only depends on the feature extraction method but also on the type of the classifier. This drives us to evaluate the performance of two different classifiers, such K-nearest neighbor algorithm (KNN), and radial basis function.

The description of each classifier models that are considered in this study are presented in the following sections.

\section{1) K-Nearest Neighbor classifier}

In pattern recognition, the k-Nearest Neighbor algorithm (k-NN) is a method for classifying objects based on closest training examples in the feature space. $\mathrm{K}-\mathrm{NN}$ is a type of instance-based learning, or lazy learning where the function is only approximated locally and all computation is deferred until classification.

The k-Nearest Neighbor algorithm is amongst the simplest of all machine learning algorithms: an object is classified by a majority vote of its neighbors, with the object being assigned to the class most common amongst its $\mathrm{k}$-Nearest Neighbors ( $\mathrm{k}$ is a positive integer, typically small). If $k=1$, then the object is simply assigned to the class of its nearest neighbor.
The same method can be used for regression, by simply assigning the property value for the object to be the average of the values of its k-Nearest Neighbors. It can be useful to weight the contributions of the neighbors, so that the nearer neighbors contribute more to the average than the more distant ones (A common weighting scheme is to give each neighbor a weight of $1 / \mathrm{d}$, where $\mathrm{d}$ is the distance to the neighbor. This scheme is a generalization of linear interpolation).

The neighbors are taken from a set of objects for which the correct classification (or, in the case of regression, the value of the property) is known. This can be thought of as the training set for the algorithm, though no explicit training step is required. The k-Nearest Neighbor algorithm is sensitive to the local structure of the data.

Nearest Neighbor rules in effect compute the decision boundary in an implicit manner. It is also possible to compute the decision boundary itself explicitly, and to do so in an efficient manner so that the computational complexity is a function of the boundary complexity [24].

The naive version of the algorithm is easy to implement by computing the distances from the test sample to all stored vectors, but it is computationally intensive, especially when the size of the training set grows. Many nearest neighbor search algorithms have been proposed over the years; these generally seek to reduce the number of distance evaluations actually performed. Using an appropriate nearest neighbor search algorithm makes k-NN computationally tractable even for large data sets.

The nearest neighbor algorithm has some strong consistency results. As the amount of data approaches infinity, the algorithm is guaranteed to yield an error rate no worse than twice the Bayes error rate (the minimum achievable error rate given the distribution of the data) [25].

K-Nearest Neighbor is guaranteed to approach the Bayes error rate, for some value of $\mathrm{k}$ (where $\mathrm{k}$ increases as a function of the number of data points). Various improvements to k-Nearest Neighbor methods are possible by using proximity graphs [26].

In summary, the k-Nearest Neighbor is a nonparametric classification procedure and hence no assumption of the form of the underlying densities is required. This method, however, assumes that there are enough points from each class such that in any small region within the decision space, the number of points occurring in these regions indicates the true nature of each density function.

\section{2) Radial basis function (RBF)}

Radial basis functions (RBFs) were originally proposed in the functional interpolation literature [27-28] and first used for discrimination by Broomhead and Lowe [29]. However, RBFs have been around in one form or another for a very long time. They are very closely related to kernel methods for density estimation 
and regression developed in the statistics literature and to normal mixture models.

The RBF may be described mathematically as a linear combination of radially symmetric nonlinear basis functions. The RBF provides a transformation of a pattern $\mathrm{x} \in \mathrm{R}^{\mathrm{p}}$ to an $n^{\prime}$-dimensional output space according to

$$
g_{j}(x)=\sum_{i=1}^{m} \omega_{j i} \phi_{i}\left(\left|x-\mu_{i}\right|\right)+\omega_{j 0}, \quad j=1, \ldots, n^{\prime}
$$

The parameters $\omega_{\mathrm{ji}}$ are often referred to as the weights; $\omega_{\mathrm{j} 0}$ is the bias and the vectors $\mu_{\mathrm{i}}$ are the centres. The model (1) is very similar to the kernel density model in which $n^{\prime}=1, \omega_{10}=0$, and the number of centres $m$ is taken to be equal to the number of data samples $n$, with $\mu_{\mathrm{i}}=\mathrm{x}_{\mathrm{i}}$ (a centre at each data sample); $\omega_{\mathrm{ji}}=1 / \mathrm{n}$ and $\phi$ is one of the kernels, sometimes referred to as the activation function in the neural network literature [30].

The above is a simple prescription for an $\mathrm{RBF}$ network that uses unsupervised techniques for centre placement and width selection (and therefore is suboptimal). One of the often-quoted advantages of the RBF network is its simplicity - there is no nonlinear optimization scheme required. However, many of the sophisticated techniques for centre placement and width determination are more involved and increase the computational complexity of the model substantially. Nevertheless, the simple RBF can give acceptable performance for many applications.

Radial basis functions are simple to construct, easy to train and find a solution for the weights rapidly. They provide a very flexible model and give very good performance over a wide range of problems, both for discrimination and for functional approximation. The RBF model uses many of the standard pattern recognition building blocks (clustering and least squares optimization, for example).

\section{RESULTS}

Two minutes of heart rate signals are shown in Fig. 3. The top and bottom panels demonstrate heart rate signals before meditation and during meditation, respectively.

According to Fig. 3, total heart rate oscillation amplitude increased and the frequency of it decreased significantly during meditation. The mean of heart rate signals also decreased during meditation (the mean of heart rate signals is about 84 beats per minute and 79 beats per minute before meditation and during meditation respectively).

Feature extraction is a critical step for classification of heart rate signals. For this purpose, in this paper spectral indices of heart rate signals during meditation and rest state are evaluated.

The power spectrum of heart rate signals for two conditions (during meditation and rest) is shown in Fig. 4.
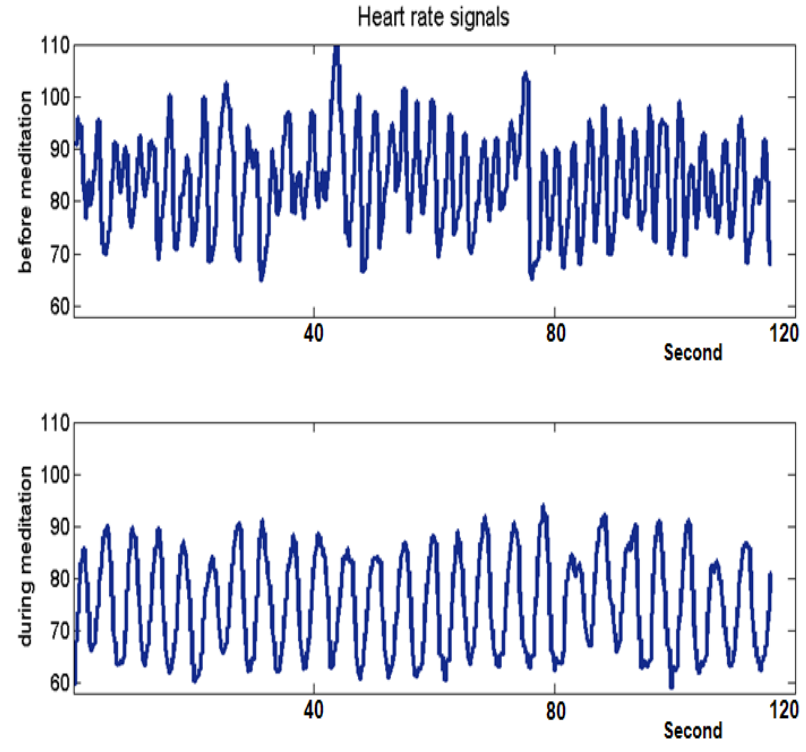

Figure 3. Heart rate signals.

Top: before Meditation; Bottom: during Meditation

Results show that the power spectrum of heart rate signals in the meditation state and the rest state are remarkably different. In addition, the LF power of heart rate signals is increased during meditation. There is a single narrowed band peak with high amplitude in the LF ranges at around $0.1 \mathrm{~Hz}$ as an example shown in Fig. 4(b).

For the rest state, there are several spectral peaks spreading throughout the frequency ranges (very low frequency; low frequency and high frequency). Furthermore, the amplitude of these components is lower than that of during meditation (as an example shown in Fig. 4(a)).

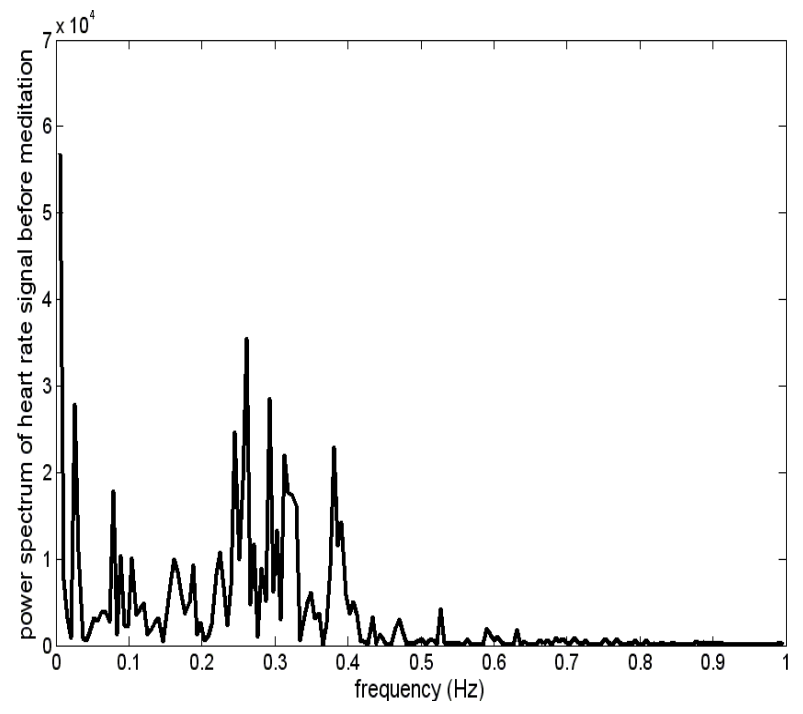

(a) 


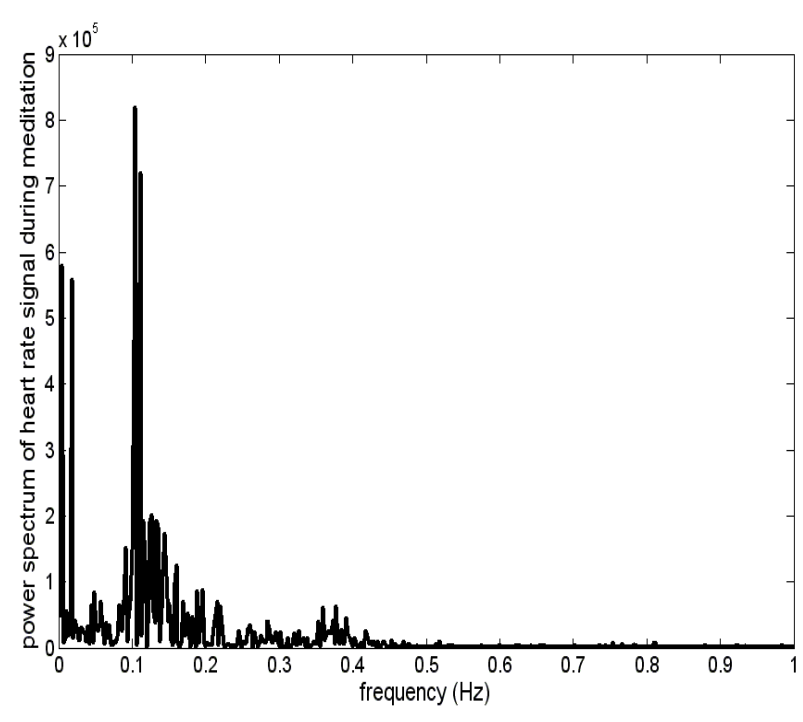

(b)

Figure 4. Power spectrum of heart rate signals.

(a) before Meditation (b) during Meditation

$\mathrm{K}-\mathrm{NN}$ classifier and RBF proposed for classification of heart rate signals were implemented by using MATLAB software package (MATLAB with PRTOOLS toolbox).

The epochs in the data set were randomly divided into two sets: a training set and a testing set. $70 \%$ of the epochs are used to train the classifiers while $30 \%$ were used to test the performance of each classifier.

For comparison of the used classifiers, the values of classification accuracy, classification error and the central processing unit (CPU) times of training of the four classifiers are presented in Table I.

TABLE I. CLASSIFICATION RESULTS.

\begin{tabular}{lccc}
\hline Classifier & $\begin{array}{c}\text { Accuracy } \\
\text { (\%) }\end{array}$ & Error & $\begin{array}{c}\text { Elapsed } \\
\text { time(s) }\end{array}$ \\
\hline $\boldsymbol{k}$ - $\boldsymbol{N N}$ & 86.5 & 0.045 & 4.2 \\
$\boldsymbol{R} \boldsymbol{B} \boldsymbol{F}$ & 92.3 & 0.026 & 5.54 \\
\hline
\end{tabular}

Note-

k-NN: k-Nearest Neighbor; RBF: Radial Basis Function

The classification results presented in Table I denote that the RBF classifier trained on spectral features obtains higher accuracy than that of k-NN classifier. The total classification accuracy of the RBF classifier is $92.3 \%$ with 0.026 classification error. As it can be observed, k-Nearest Neighbor classifier gives encouraging results $(86.5 \%)$.

\section{IV.DISCUSSION}

This paper presented a new application of classifiers employing spectral features (very low frequency; low frequency; high frequency; the LF/HF ratio and frequency of the highest spectral peak of heart rate signals) for classification of the heart rate signals during rest and meditation.

It has shown that total heart rate oscillation amplitude increased and the frequency of it decreased significantly during meditation (Fig. 3). In addition, the mean of heart rate signals decreased during meditation (the mean of heart rate signals is about 84 beats per minute and 79 beats per minute before meditation and during meditation respectively).

The variations in heart rate can be separated into different components by use of spectral analysis. The frequency domain analysis of heart rate signals reveals that the pattern of the power spectrum is remarkably different in the meditation state than that of the rest state. In addition, the LF power of heart rate signals is increased during meditation. There is a single narrowed band peak with high amplitude in the LF ranges at around $0.1 \mathrm{~Hz}$ as an example shown in Fig. 4(b), but for the rest state, there are several spectral peaks spreading throughout the frequency ranges. Furthermore, the amplitude of these components is lower than that of during meditation (as an example shown in Fig. 4(a)). These results were similar to our previous meditation studies [21,31].

As the low frequency components of heart rate signals are dominant, we can conclude that heart rate signals are affected by both the sympathetic and parasympathetic systems during meditation.

In the present study, k-Nearest Neighbor (k-NN) and Radial Basis Function (RBF) are used to classify two states of heart rate signals, rest and meditation, when the spectral indices of heart rate signals are used as the classifiers inputs.

As the classifier performance depends on the features extraction to represent each class, the result of present study indicated that spectral indices are efficient in classification of heart rate signals in two states: rest and meditation.

The results indicate that the classification of heart rate signals (rest and meditation states) using $\mathrm{RBF}$ classifier trained on spectral features obtains higher accuracy than that of k-NN classifier (Table I). According to the results, the total classification accuracy of the RBF classifier is $92.3 \%$ with 0.026 classification error; while the accuracy of k-Nearest Neighbor classifier is $86.5 \%$ with 0.045 classification error.

\section{CONCLUSION}

Results of current study prove that most of heart rate power is concentrated in the low frequency bands which reflect that heart rate signals are affected by both the sympathetic and parasympathetic systems during meditation.

For comparison of different classifiers, we implemented the k-Nearest Neighbor and Radial Basis Function to deal with the same classification and each of them is used to handle heart rate classification based on a specific feature set. 
The classification accuracies, errors and the CPU times of training showed that the RBF classifier trained on the five feature vectors (very low frequency; low frequency; high frequency; the LF/HF ratio and frequency of the highest spectral peak of heart rate signals) produce considerably good performance. The obtained results demonstrated that the proposed classifier can be used in classification of the heart rate signals during meditation and rest.

Heart rate signals have valuable information about physiological changes during meditation; however, more studies can be carried out on classification of other signals such as electroencephalograms and respiration rates.

\section{ACKNOWLEDGEMENTS}

We thank Dr. Minoo Morvarid, the Master of Meditation Clinic in Mashhad, and Ms. Shahla Khoshkholgh for their assistance in collecting the data that were used in the investigations. The authors would also like to thank all the subjects for their good collaborations.

\section{REFERENCES}

[1] Walsh R, Shapiro SL. (2006). The meeting of meditative disciplines and Western psychology: a mutually enriching dialogue. Am Psychol, 61(3), 227-239.

[2] Tang YY, Ma Y, Wang J, Fan Y, Feng S, Lu Q, et al. (2007). Short-term meditation training improves attention and self-regulation. Proceeding of the National Academy of Science USA, 104, 17152-17156.

[3] Matousek RH, Dobkin PL, Pruessner J. (2010). Cortisol as a marker for improvement in mindfulness-based stress reduction, Complementary Therapies in Clinical Practice, 16, 13-19.

[4] Grossman P, Niemann L, Schmidt S, et al. (2004). Mindfulness-based stress reduction and health benefits: a meta-analysis. J Psychosom Res, 57(1), 35-43.

[5] Shapiro SL, Astin J, Bishop S, Cordova M. (2005). Mindfulness-based stress reduction for health care professionals: Results from a randomized trial. International Journal of Stress Management, 12(2), 164-176.

[6] Goshvarpour A., Goshvarpour A., Rahati S. (2011). Analysis of lagged Poincare plots in heart rate signals during meditation, Digital Signal Processing, 21, 208-214.

[7] Maestri R, Rovere MT La, Porta A, Pinna GD. (2008). Sympathetic Neurohormonal Correlates of Linear and Symbolic Dynamics Heart Rate Variability Indexes in Chronic Heart Failure, Computers in Cardiology, 35, 49-52.
[8] Vandeput S., Verheyden B., Aubert A.E., Huffel S.V. (2008). Nonlinear heart rate variability in a healthy population: influence of age, Computers in Cardiology, 35, 53-56.

[9] Goshvarpour A., Goshvarpour A. (2012). Chaotic Behavior of Heart Rate Signals during Chi and Kundalini Meditation. I.J. Image, Graphics and Signal Processing, 2, 23-29.

[10] Goshvarpour A., Goshvarpour A. (2012). Recurrence Plots of Heart Rate Signals during Meditation, I.J. Image, Graphics and Signal Processing, 2, 44-50.

[11] Lehrer P, Sasaki Y, Saito Y. (1999). Zazen and cardiac variability, Psychosomatic Medicine, 61, 812-821.

[12]Berntson GG, Bigger JT Jr, Eckberg DL, Grossman P, Kaufmann PG, Malik M, Nagaraja HN, Porges SW, Saul JP, Stone PH, van der Molen MW. (1997). Heart rate variability: origins, methods, and interpretive caveats. Psychophysiology, 34, 623648.

[13] Task Force of the European Society of Cardiology and the North American Society of Pacing and Electrophysiology. (1996). Heart rate variability: standards of measurement, physiological interpretation, and clinical use. Circulation, 93, 1043-65.

[14]Porges SW, Bohrer RE. (1990). Analysis of periodic processes in psychophysiological research. In: Caccioppo JT, Tassinary LG, editors. Principles of psychophysiology: physical, social, and inferential elements. Cambridge: Cambridge University Press, 708-753.

[15] Berntson GG, Caccioppo JT, Quigley KS. (1993). Respiratory sinus arrhythmia: autonomic origins, physiological mechanisms, and psychophysiological implications. Psychophysiology, 30, 183-96.

[16] Porges SW. (1992). Vagal tone: physiologic marker of stress ulnerability. Pediatrics, 90, 498-504.

[17] Malliani, A., M. Pagani, F. Lombardi, and S. Cerutti. (1991). Cardiovascular neural regulation explored in the frequency domain. Circulation 84, 482-492.

[18]Bernardi L, Leuzzi S, Radaelli A, Passino C, Johnston JA, Sleight P. (1994). Low frequency spontaneous fluctuations of R-R interval and blood pressure in conscious humans: a baroreceptor or central phenomenon? Clin Sci, 87, 647-54.

[19] Takahashi T, Murata T, Hamada T, Omori M, Kosaka H, Kikuchi M, Yoshida H, Wada Y. (2005). Changes in EEG and autonomic nervous activity during meditation and their association with personality traits, International Journal of Psychophysiology, 55, 199-207.

[20] Taylor JA, Carr DL, Meyers CW, Eckberg DL. (1998). Mechanisms underlying very-lowfrequency RR-interval oscillations in humans. Circulation, 98, 547-55.

[21] Goshvarpour A., Rahati S., Saadatian V. (2010). 
Estimating depth of meditation using electroencephalogram and heart rate signals [MSc. Thesis] Department of Biomedical Engineering, Islamic Azad University, Mashhad Branch, Iran. [Persian]

[22] Goshvarpour A., Rahati S., Saadatian V. (2010). Analysis of electroencephalogram and heart rate signals during meditation using Hopfield neural network [MSc. Thesis] Department of Biomedical Engineering, Islamic Azad University, Mashhad Branch, Iran. [Persian]

[23] Cerutti S, Biachi AM, Mainardi LT. (1995). Spectral analysis of the heart rate variability signal. In: Malik M, Camm AJ, editors. Heart rate variability, New York: Futura, 63-74.

[24] Bremner D., Demaine E., Erickson J., Iacono J., Langerman S., Morin P., Toussaint G. (2005). Outputsensitive algorithms for computing nearestneighbor decision boundaries. Discrete and Computational Geometry, 33(4), 593-604.

[25] Cover T.M., Hart P.E. (1967). Nearest neighbor pattern classification, IEEE Transactions on Information Theory, 13(1), 21-27.

[26] Toussaint G.T. (2005). Geometric proximity graphs for improving nearest neighbor methods in instance-based learning and data mining. International Journal of Computational Geometry and Applications, 15(2), 101-150.

[27] Powell, M.J.D. (1987). Radial basis functions for multivariable interpolation: a review. In J.C.Mason and M.G. Cox, eds, Algorithms for Approximation, 143-167. Clarendon Press, Oxford.

[28]Lowe, D. (1995). Radial basis function networks. In M.A. Arbib, ed., The Handbook of Brain Theory and Neural Networks, 779-782. MIT Press, Cambridge, MA.

[29] Broomhead, D.S. and Lowe, D. (1988). Multivariable functional interpolation and adaptive networks. Complex Systems, 2(3), 269-303.

[30]M. Andrew R. Webb . QinetiQ Ltd., UK, (2002). Statistical Pattern Recognition. England,

[31] Goshvarpour A., Goshvarpour A., and Rahati S. Analysis of heart rate signals during meditation. (2010). The $2^{\text {nd }}$ National Electrical Engineering Conference, Najafabad Branch of IAU. [Persian]

Ateke Goshvarpour: Obtained a Masters in Biomedical Engineering from Islamic Azad University, Mashhad Branch, Iran in 2010. Her thesis research focused on analyzing biomedical signals during meditation. In addition, her research interests include biomedical signal processing, mathematical modeling, nonlinear analysis and neural networks.

Atefeh Goshvarpour: Obtained a Masters in Biomedical Engineering from Islamic Azad University, Mashhad Branch, Iran in 2010. Her thesis research focused on analyzing biomedical signals during meditation. In addition, her research interests include biomedical signal processing, mathematical modeling, nonlinear analysis and neural networks. 